\title{
Long Term Effects of Metformin in a Non-Diabetic Population with Differentiated Thyroid Carcinoma
}

\author{
Monti Eleonora $^{1 *}$, Balestra Margherita ${ }^{1}$, Minuto N Michele ${ }^{2}$, Gay Stefano ${ }^{1}$, Conte Lucia ${ }^{1}$ and Giusti Massimo ${ }^{1}$ \\ ${ }^{1}$ Department of Internal Medicine (Di.M.I.) University of Genoa and IRCSS Ospedale Policlinico San Martino, Genoa
}

${ }^{2}$ Department of Surgical Sciences (DISC) University of Genoa and UO Chirurgia IRCCS Ospedale Policlinico San Martino, Genoa

Received: October 10,2019; Accepted: October 22,2019; Published: October 30,2019

*Corresponding author : Monti Eleonora, Department of Internal Medicine (Di.M.I.) University of Genoa and IRCSS Ospedale Policlinico San Martino, Genoa, Italy. Email: eleonora.monti87@gmail.com

\begin{abstract}
Introduction: Differentiated thyroid carcinoma (DTC) is the most frequent endocrine neoplasm. Type 2 diabetes mellitus (DMT2) and cancer share several risk factors. Metformin is used as an anti-hyperglycemic agent to reduce insulin resistance. Its anti-proliferative role is widely discussed in the literature.

Materials and Methods: 95 DTC patients without a diagnosis of DMT2 (group 1), in whom metformin was started, 79 non-diabetics DTC patients who did not start metformin (group 2) served as a control group. All subjects were evaluated at the baseline and after 12 and 24 months. Group 1 was also evaluated at 3 months and 36 month to assess the tolerability of metformin and then the therapy compliance.

Results: Prospective evaluation of group 1 versus group 2: no differences in the values of TSH at 24 months ( $\mathrm{P}=0.23)$; no differences in L-T4 dosages. No significant difference in Tg values emerged between group 1 and group $2(P=0.06)$, nor between baseline Tg and the values at $3(P=$ 0.06), $12(\mathrm{P}=0.1), 24$ months $(\mathrm{P}=0.25)$ after the start of metformin. No differences in group 1 in the values of $\operatorname{Tg}(\mathrm{P}=0,79)$ or $\mathrm{TSH}(\mathrm{P}=0.26)$ between baseline and 36 months. In group 1, a significant difference was found in total cholesterol levels between the baseline and the 3-month ( $\mathrm{P}=0.01$ ), 12-month $(\mathrm{P}=0.0001), 24$-month $(\mathrm{P}=0.005)$ and 36 months-examinations $(\mathrm{P}=0.0001)$; total cholesterol levels progressively declined in group 1 , and a significant difference emerged between group 1 and group 2 at $12(\mathrm{P}=0.036)$ and 24 months $(\mathrm{P}=0.01)$.
\end{abstract}

Conclusions: The present study has the most numerous DTC population without DM in whom metformin has been started as off-label therapy. Metformin did not seem to have an effect on TSH. Metformin reduced total and LDL cholesterol levels, yielding a possible cardiovascular advantage. $\mathrm{Tg}$ data were inconclusive, perhaps because this population has a very good prognosis after primary treatments.

Keywords: Differentiated thyroid carcinoma; Metformin; Cancer.

\section{Introduction}

Differentiated thyroid carcinoma (DTC) arising from follicular cells is the most frequent endocrine neoplasm and is diagnosed in $5 \%$ of thyroid nodules [1]. Type 2 diabetes mellitus (DMT2) and cancer share some potential risk factors [2]. The incidence of DTC in diabetes mellitus has not been thoroughly studied and the results seem inconclusive, as in the case of other types of cancer [3]. Metformin seems to reduce the risk of DTC 2-3-fold in DMT2 [4]. Metformin activates AMPc-dependent protein kinase (AMPK) in the liver. Metformin is a positively charged molecule that targets mitochondrial complex 1 and the formation of ATP: this effect can influence different pathways in many organs and tissues [5]. It has been suggested that reducing ATP contributes to the inhibition of gluconeogenesis, and that this response could also be independent of AMPK [6]. Another study demonstrated that metformin non-competitively inhibits the enzyme glycerophosphate dehydrogenase, with a consequent reduction in gluconeogenesis [7]. The gluconeogenesis induced by glucagon can be inhibited by metformin through the inhibition of AMPc production and of PKA activity. Clinical and epidemiological studies support the role of metformin in reducing the overall risk of carcinoma and disease-dependent mortality, but the effects seem to vary according to the type of carcinoma [8], and the mechanism of action is not fully understood. Metformin works indirectly by reducing blood glucose levels and blocking insulin- and IGF-1-dependent signals, which lead to the inhibition of tumour growth. It also operates through a direct mechanism, activating the AMPK pathway and, consequently, inhibiting mTOR $[9,2,8]$; specifically, when cellular ATP levels decrease, the complex LKB1 (a well-known tumour suppressor) - AMPK is activated, leading to the inhibition of mTOR. MTOR is also inhibited by blockage of PI3K (insulin-activated kinase, growth factors, cytokines); this slows down protein and glucose synthesis in the liver, thereby reducing the bioavailability of energy and nutrients and hindering tumour proliferation [2]. With specific reference to DTC, clinical trials have shown that treatment with metformin is associated with a higher remission rate and longer survival in diabetic patients with thyroid cancer [10] and with a more favourable outcome in subjects with lymph 
node metastases [11]. A large observational study in Taiwan showed that DMT2 patients treated with metformin had a lower risk of thyroid cancer [12]. In the last decade, several studies have shown that metformin reduces TSH levels in hypothyroid patients with DMT2 and polycystic ovary, without altering the circulating levels of thyroid hormones, regardless of thyroid autoimmunity or hormone therapy. It has been hypothesized that metformin can increase the action of hormones at the central level, though a concomitant peripheral effect has not been excluded [13] In peripheral circulation metformin activates AMPK, while in the hypothalamus, metformin suppresses AMPK [14]. Krysiak et al. [15] conducted a study on Polycystic Ovary Syndrome's (PCOS) patients, 12 of whom were treated with bromocriptine; after 6 months of treatment with metformin, the TSH-reducing effect was greater in subjects not treated with bromocriptine. This result could indicate that the action of metformin is partly associated with a change in dopaminergic tone. Other hypotheses include: a change in the affinity of $\mathrm{T} 3$ and $\mathrm{T} 4$ receptors, a change in their transporter proteins, or a change in their metabolism and bioavailability, inducing a constitutive receptor activation. It can be ruled out that metformin influences the absorption of L-T4, as suggested in early studies: indeed, most studies have found no concomitant modification of $\mathrm{f}-\mathrm{T} 4$ or $\mathrm{f}-\mathrm{T} 3$ in response to metformin. Al-Alusi et al. [16] also confirmed that there was no evidence of a change in L-T4 absorption with the concomitant intake of metformin and, in addition, found in TSH even in hypothyroid patients not treated with L-T4.

\section{Purpose of the Study}

The aim of the present study was to prospectively evaluate the long term effects of metformin in a non-diabetic population of DTC patients. The study was conducted during the periodic monitoring (2013-2016) that DTC patients underwent at the Thyroid Oncology Outpatient Clinic of the Endocrinological Clinical Unit. The main objective was to verify the effectiveness of metformin in controlling thyroid disease in DTC. The secondary objectives were: to verify compliance with further, currently off-label, therapy in non-diabetic chronic patients, and to verify the effectiveness of metformin on the hormonal and metabolic profile, with a view to remotely implementing changes to therapy, such as reducing the L-T4 dose, in order to avoid the risk of side effects typical of hormone treatment (e.g. anxiety, arrhythmias and bone resorption) and to achieve better control of metabolic comorbidities concomitant with DTC.

\section{Materials and Methods}

\section{a) Subjects}

We recruited 95 DTC patients without a diagnosis of type 2 diabetes mellitus (DMT2) (group 1) in which metformin was started. Our control group consisted of 79 non-diabetic DTC patients who were not on metformin (group 2). In the period 2013-16, the 95 DTC non-diabetic patients were offered treatment with metformin as an adjuvant to L-T4 in the control of post-surgical hypothyroidism and as a potential anti-proliferative drug. All patients were adults; women of reproductive age were considered for metformin treatment only if they expressed a current intention not to become pregnant. Patients with known chronic renal insufficiency (GFR $<30 \mathrm{ml} / \mathrm{min}$ ) were excluded. Patients were randomly selected for enrolment (1 every 2-3 who reached the evaluation phase) from among all the nondiabetic DTC patients attending the Outpatient Clinic for Thyroid Oncological Pathology of the Endocrinological Clinical Unit. The effects of metformin were explained in advance to all patients and the drug was presented as an anti-diabetic that does not cause hypoglycaemia, as it reduces insulin resistance, if present. All patients were shown a comprehensible summary of the literature, and received an information letter for their general practitioner, explaining the reason for their inclusion in the study in the absence of chronic hyperglycaemia. Patients were informed in advance about the potential and most common side effects of the drug e.g. bowel sounds, flatulence, nausea, abdominalgia. Of the 95 DTC patients selected for metformin treatment, 7 were lost to follow-up, 15 patients discontinued the drug owing to side effects, and 3 withdrew their consent; these subjects were excluded from the statistical evaluation. Thus, the group of non-diabetic DTC patients treated with metformin consisted of 70 DTCs (group 1). The study protocol was presented to the Ethics Committee of the Policlinico San Martino of Genoa, and all participating patients provided written informed consent.

\section{b) Protocol}

All patients were evaluated at the start of the study. The patients in each group underwent a preliminary basal test and patients in group 1 also underwent an interim evaluation 3 months after starting metformin, in order to assess the tolerability and effectiveness of the drug. In group 1, metformin was started at a dosage of $250 \mathrm{mg} /$ day; if the drug was well tolerated, the dosage was increased by $250 \mathrm{mg}$ steps to $1000 \mathrm{mg}$ / day. At each evaluation, patients who confirmed their informed consent underwent: a) anamnesis, with particular attention to subjective symptoms related to the therapy, and evaluation of L-T4 intake; b) physical examination of the neck and whole body, and measurement of systolic and diastolic blood pressure (PA $\mathrm{mmHg}$ ), heart rate and body mass index (BMI kg/m2); c) blood sampling; d) neck ultrasound.

\section{c) Laboratory evaluations}

Blood samples were taken in the fasting state between 8 am and $10 \mathrm{am}$. A biochemical test was carried out to evaluate free thyroid hormones, thyrotropin (TSH), thyroglobulin (Tg) and anti-Tg antibodies (TgAb). When available, the result of human recombinant (rh)-TSH testing was considered for an further information. The biochemical test was performed in the standard mode (Thyrogen Genzyme, $0.9 \mathrm{mg}$ IM on two consecutive days) with Tg detections 0, 3, 6 and 9 days after the stimulus - combined or not with total-body 131I scintigraphy [17]. As part of a more extensive clinical chemistry assessment, the following parameters were also evaluated in the fasting state: glycaemia, glycosylated haemoglobin (HbA1c), insulin, creatinine, cholesterol, HDL cholesterol and triglycerides. A glycosylated haemoglobin value $>6.5 \%$ ( $>48 \mathrm{mmol} / \mathrm{mol}$ ) is taken as the threshold value for the diagnosis of diabetes (Italian Standards for the treatment of diabetes mellitus, 2018). LDL-cholesterol levels were calculated according to the formula of Friedewald [18] [LDL cholesterol 
$=$ total cholesterol $-($ HDL cholesterol + triglycerides $/ 5)]$. This formula was not applied in subjects with triglyceride levels $>240 \mathrm{mg} / \mathrm{dl}$. Insulin resistance was calculated according to the algorithm of the HOMA index (Homeostasis Model Assessment) [glycaemia (mmol/) l x insulinemia $(\mu \mathrm{U} / \mathrm{ml})$ ] / 22.5] [19].

\section{d) Assays}

The serum concentration of $\mathrm{Tg}$ was determined by means of chemiluminescence immunoassay (Roche Diagnostics). The assay was standardised by using the CRM 475 standard of the Community Bureau of References of the European Commission. The functional sensitivity is $<0.1 \mathrm{ng} / \mathrm{ml}$. The intra- and inter-test coefficient of variation (CV) was less than $5-8 \%$. On the basis of functional sensitivity, the value of $0.1 \mathrm{ng} / \mathrm{ml}$ was chosen as the cut-off between the determinable and indeterminable Tg levels. $\mathrm{TgAb}$ was determined by means of a commercial assay (Radim) with a cut-off value of $100 \mathrm{U} / \mathrm{L}$. In series with TgAb levels $>100$ $\mathrm{U} / \mathrm{L}, \mathrm{Tg}$ was determined after recovery testing. TSH and free thyroid hormones were evaluated by using an ultra-sensitive chemiluminescence method (Roche Diagnostics). Normal values were: 0.3-4.2 mU/L for TSH; $2.7-7.0 \mathrm{pmol} / \mathrm{L}$ for free T3 (f-T3); and 11.5-21.8 pmol/L for free T4 (f-T4). Insulin was evaluated by means of chemiluminescent immunoassay (DENKA SEIKEN, Tokyo Japan); the $\mathrm{cv}$ was $4 \%$ for $7.6 \mu \mathrm{U} / \mathrm{mL}$ insulin values and $5 \%$ between tests. The sensitivity of the method is equal to $1 \mu \mathrm{U} / \mathrm{mL}$. Creatinine serum levels (normal values $<1.2 \mathrm{mg} / \mathrm{dl}$ ), blood sugar (normal values: $60-110 \mathrm{mg} / \mathrm{dl}$ ), total cholesterol (130-200 mg/ dl), HDL cholesterol ( $>60 \mathrm{mg} / \mathrm{dl})$ and triglycerides $(<150 \mathrm{mg} /$ dl) were evaluated by means of fully automatic Modular P800 (Roche). Glycosylated haemoglobin (HbA1c) was determined by means of automatic liquid chromatography equipment HLC723G7 TOSOH Co. in whole blood collected in tubes containing sodium citrate. The data were expressed as percentages of the normal haemoglobin value, with a range of $4.3-5.8 \%$.

\section{e) Statistical analysis}

During follow-up examinations, the effectiveness of primary treatments (thyroidectomy, ablation with radioactive iodine (RAI) therapy) was evaluated on L-T4 therapy at dosages that were TSH-suppressive or semi-suppressive, depending on the initial stratification of risk. In the presence of negative $\mathrm{TgAb}$ antibodies, undetectable $\mathrm{Tg}$ levels on TSH suppression - or detectable only after stimulation with rhTSH - together with negative ultrasound imaging (US) of the neck, were considered to indicate complete remission (CR) of DTC. When available, totalbody 131-I scintigraphy (WBS), computed tomography (CT) and positron emission tomography (PET/TC) were used as additional elements of assessment. Biochemical disease persistence was defined by detectable Tg levels in DTC patients who had undergone total thyroidectomy and RAI, with no determinable structural lesions. A high Tg level and positive imaging were elements for considering indicated that DTC was persistent or recurrent. Continuous variables were compared by means of non-parametric statistical tests. Correlation between 2 variables was assessed by means of the Mann-Whitney test, while the Wilcoxon test was used to compare parameters within the same population and the Kruskal-Wallis test to compare data among different populations. In all tests, a $\mathrm{P}$ value $<0.05$ was taken to be significant. Statistical analyses were carried by means of the MedCalc 15.8 programme.

\section{Results}

\section{a) Basal test}

The populations were comparable in terms of histology of the tumour, the stage of the disease and the mode of surgical treatment after the diagnosis (Table 1). Groups 1 and 2 were not significantly different in age $(\mathrm{P}=0.8)$. Group 1 differed significantly from group 2 in terms of gender composition, in

Table 1: Basal test in the groups analysed; the data are shown as average values \pm SE (standard error)

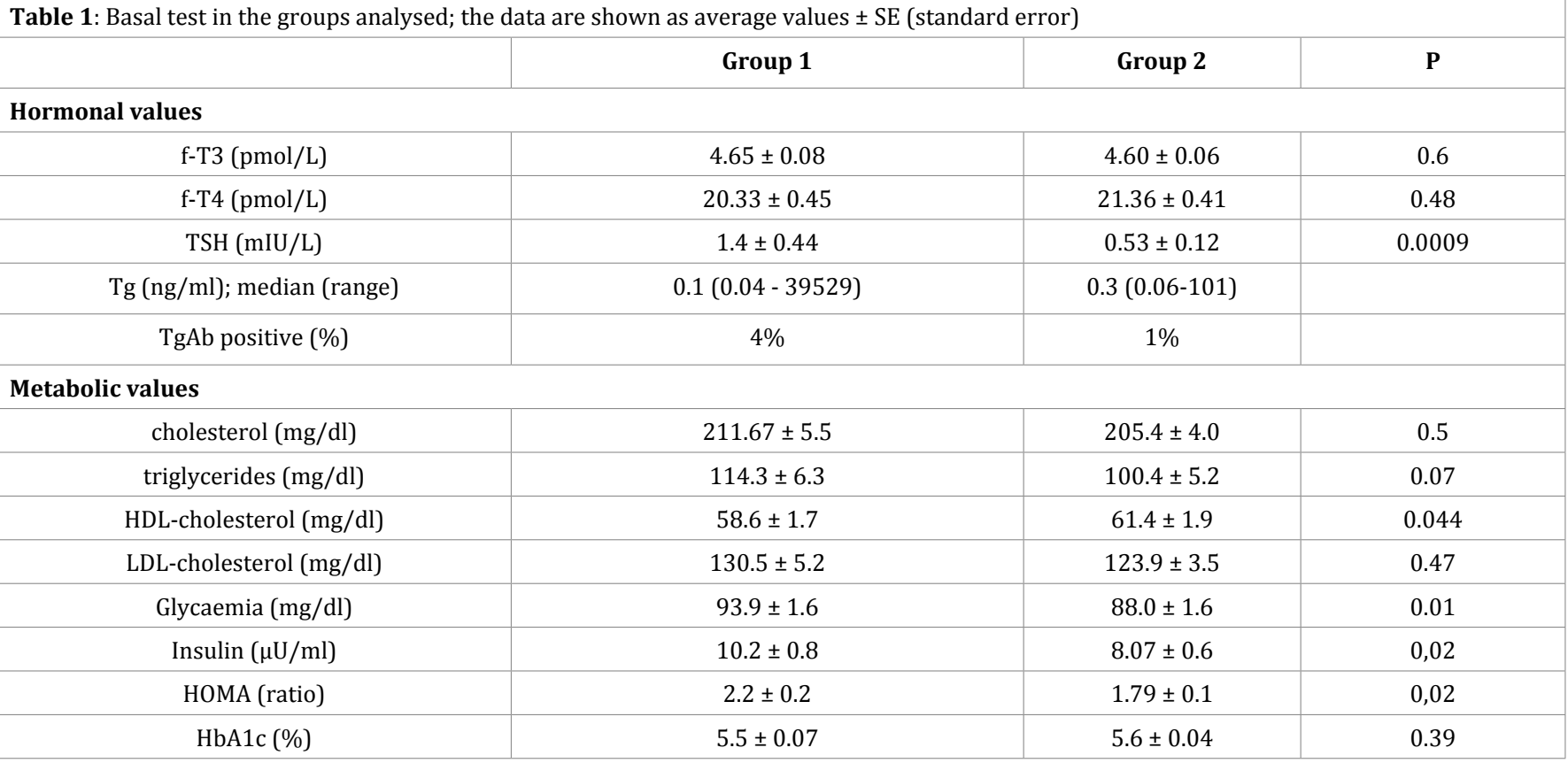


that fewer group 1 patients were female $(\mathrm{P}=0.03)$. With regard to hormonal parameters, a statistically significant difference was observed between basal Tg values in group 1 and group 2 $(\mathrm{P}=0.0004)$, with higher $\mathrm{Tg}$ values in group 1 . TSH values were significantly higher in group 1 than in group $2(\mathrm{P}=0.0009)$. BMI was significantly higher in group 1 than in group $2(\mathrm{P}=0.0003)$. No significant differences were seen among groups in total cholesterol $(\mathrm{P}=0.15)$ or LDL cholesterol $(\mathrm{P}=0.16)$ or triglyceride values $(\mathrm{P}=0.07)$ at baseline. No statistically significant difference emerged between group 1 and group $2(\mathrm{P}=0.39)$ in glycated haemoglobin values but fasting glycaemia was higher in group 1 than group $2(\mathrm{P}=0.01)$. Insulinemia values were higher in group 1 than in group $2(\mathrm{P}=0.02)$, HOMA values were higher in group 1 than in group $2(\mathrm{P}=0.026)$. In figures $1-2$ there is represented risk stratification of groups according to American Thyroid Association (ATA) guidelines [1].

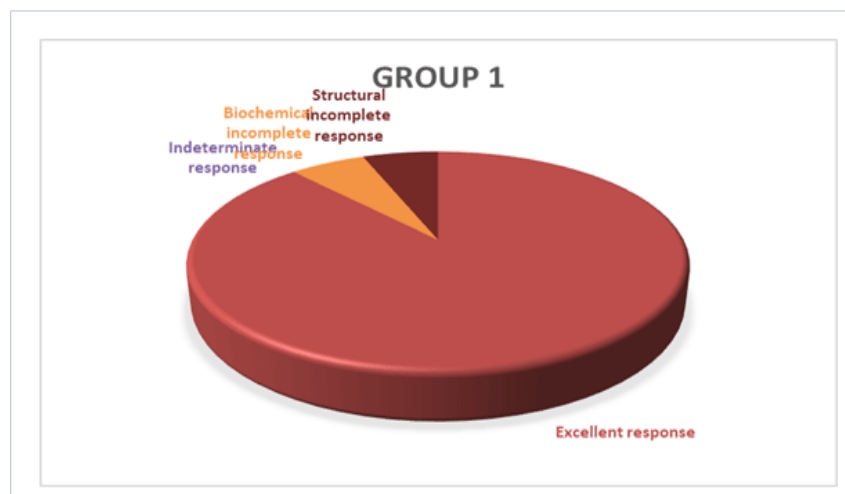

Figure 1: Group 1 risk stratification according to the ATA 2015 guidelines

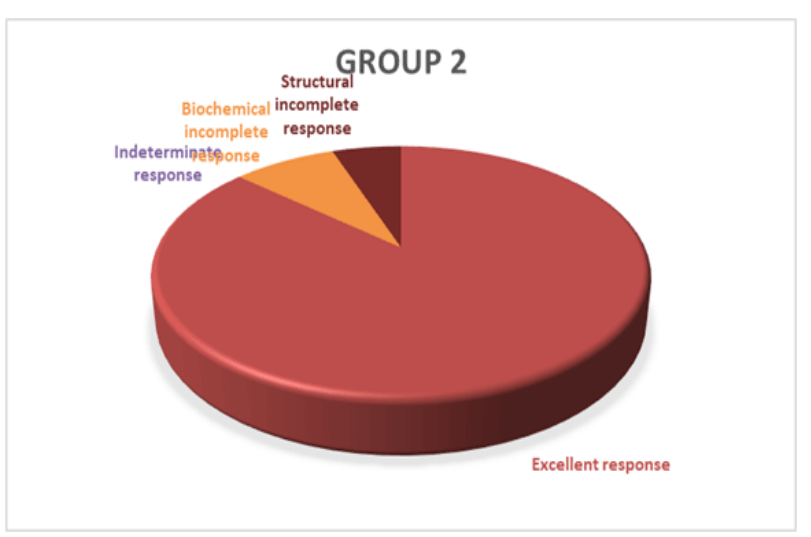

Figure 2: Group 2 risk stratification according to ATA 2015 guidelines

\section{b) Controlled prospective evaluation of DTC patients without DMT2 treated with metformin}

Of the 95 group 1 patients who started the prospective evaluation, 7 were lost to follow-up, 15 discontinued the drug owing to side effects, and 3 withdrew their consent. Thus, hormonal and metabolic data were available at the 3-month follow-up examination in 70 patients on metformin. Patients were evaluated 3,12, 24 and 36 months after the start of metformin. At 12 months, 2 patients did not attend for examination, and 2 patients had discontinued the drug owing to side effects (hence, full 12-month data on 66 patients were available). At 24 months, 5 patients had discontinued the drug owing to non-specific intolerance, 2 patients have yet to be tested after 24 months, and 2 patients have died (hence, full 24-month data on 55 patients were available). At 36 months 2 patients had discontinued the drug, 1 patient has died and 1 was discontinued the follow-up (hence, full 36-month data on 51 patients were available). In 15 patients, the recommended dose of metformin $(1000 \mathrm{mg} /$ day $)$ was not reached. Figure 3 reports the side effects of metformin. Regarding positive effects, two patients experienced weight loss and their previous constipation ceased. During the study, creatininemia data (expressed as average $\pm \mathrm{SE}$ ) were normal: $0.84 \pm 0.02 \mathrm{mg} / \mathrm{dl}$ in group 1 and $0.81 \pm 0.02 \mathrm{mg} / \mathrm{dl}$ in group 2, both at 24 months. There were no differences between group 1 and control group $2(\mathrm{P}=0.4)$.

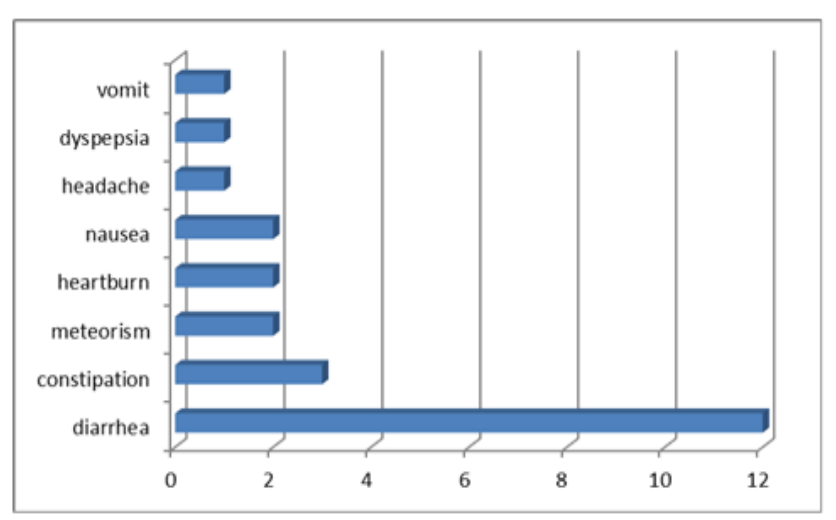

Figure 3: Number and type of adverse events reported by DTC patients without DMT2 (group 1) on metformin treatment

BMI was significantly higher in group 1 than in group 2 (P $=0.009)$; this finding was confirmed after 12 months $(\mathrm{P}=0.008)$ and 24 months $(\mathrm{P}<0.0001)$.

Blood pressure and heart rate were controlled in most in group 1 subjects, without substantial changes during the study: systolic ( $\mathrm{P}=0.29$ at 12 months; $\mathrm{P}=0.95$ at 24 months) and diastolic ( $\mathrm{P}$ $=0.06$ at 12 months and $\mathrm{P}=0.25$ at 24 months); after 12 and 24 months on metformin, blood pressure was not significantly different from the basal value, nor were any differences in heart rate found ( $\mathrm{P}=0.24$ at 12 months; $\mathrm{P}=0.07$ at 24 months).

In group 1, TSH was higher at the baseline than on 3-month evaluation $(\mathrm{P}=0.0045)$; this finding was confirmed at 12 months $(\mathrm{P}=0.01)$, but not at 24 months $(\mathrm{P}=0.23)$ nor at 36 months $(\mathrm{P}=0.79)$. Regarding the L-T4 dosage administered, no significant differences were observed in group 1 between the basal test, the 12-month $(\mathrm{P}=0.53)$, the 24 -month $(\mathrm{P}=0.73)$ and 36 months $(\mathrm{P}=0.48)$ follow-up examinations. No statistically significant differences in F-T4 were found between the basal test and the 3-month $(\mathrm{P}=0.36), 12$-month $(\mathrm{P}=0.01)$ and 24-month $(\mathrm{P}=0.15)$ evaluations. 
At the baseline, TSH was higher in group 1 than in group 2 $(\mathrm{P}=0.0009)$. The same pattern was seen at the 12 -month followup examination, though the significance was lower $(\mathrm{P}=0.02)$. At the 24-month assessment, no statistically significant differences emerged ( $\mathrm{P}=0.81)$. At the 24-month follow-up examination, the total dose of LT4 was significantly higher in group 1 than in group $2(\mathrm{P}=0.01)$, while there was no significant difference between these two groups at the baseline $(\mathrm{P}=0.05)$.

In group 1, BMI was lower at 12 months than in the basal test $(\mathrm{P}=0.008)$ but no statistically significant differences emerged between baseline and 24-months $(P=0.62)$ nor 36 months $(\mathrm{P}=0.23)$ values. $\mathrm{BMI}$ was confirmed to be higher in group 1 than in group 2 at $12(\mathrm{P}=0.008)$ and $24(\mathrm{P}=0.001)$ months. Regarding Tg data, no significant differences were found between group 1 and group $2(\mathrm{P}=0.06)$ nor between basal thyroglobulin and 3-month $(\mathrm{P}=0.06), 12$-month $(\mathrm{P}=0.1)$ or 24 -month $(\mathrm{P}=0.25)$ values in group 1.

Glycated haemoglobin was lower in group 1 than in group 2 at 12 months $(P=0.039)$ but not at 24 months $(P=1.0)$. In group 1 , glycated haemoglobin was higher at the baseline than at 3 months $(\mathrm{P}=0.02), 12$ months $(\mathrm{P}=0.009)$ and 24 months $(\mathrm{P}=0.02)$.

Fasting glycaemia was higher at the baseline that at 3 months $(\mathrm{P}=0.001)$ and 12 months $(\mathrm{P}=0.0018)$, but not 24 months $(\mathrm{P}$ $=0.15)$, while no statistically significant differences in fasting glucose were found between group 1 and group 2 at 12 months $(\mathrm{P}=0.76)$ or 24 months $(\mathrm{P}=0.57)$.

No difference in the values of insulinemia emerged between the baseline and the 3-month $(\mathrm{P}=0.31), 12$-month $(\mathrm{P}=0.46)$ or 24-month ( $\mathrm{P}=0.79)$ evaluations in group 1 ; in this group, insulinemia was higher than in group 2 at 12 months $(\mathrm{P}=0.02)$ and at 24 months $(\mathrm{P}=0.05)$.

In group 1, the HOMA index did not differ significantly between the basal test and the follow-up evaluations at 3 months $(\mathrm{P}=0.16), 12$ months $(\mathrm{P}=0.17)$ and 24 months $(\mathrm{P}=0.95)$. However, a higher HOMA index was found in group 1 than in group 2 at 12 months $(P=0.04)$ but not at 24 months $(P=0.21)$.

No significant changes in renal function were observed in group 1 between the basal test and the 24-month evaluation (P $=0.75)$; moreover, no difference in creatinine between group 1 and group 2 was observed at 12 months $(\mathrm{P}=0.26)$ or 24 months $(\mathrm{P}=0.41)$.

Total cholesterol levels in group 1 progressively declined, displaying significant differences between the basal test and the 3-month $(\mathrm{P}=0.01), 12$-month $(\mathrm{P}=0.0001), 24$-month $(\mathrm{P}=0.005)$ and 36 month $(\mathrm{P}=0.0001)$ evaluations. Total cholesterol levels were significantly lower in group 1 than in group 2 at $12(\mathrm{P}=$ 0.036) and 24 months $(\mathrm{P}=0.01)$.

With regard to triglycerides levels, no significant differences were found between the basal test and the 3-month $(\mathrm{P}=0.65)$, 12-month $(\mathrm{P}=0.05)$ and 24-month $(\mathrm{P}=0.61)$ evaluations in group 1 . At 24 months, triglyceride values were higher in group 1 than in group $2(\mathrm{P}=0.04)$, though no difference emerged between these groups at the 12-month evaluation $(\mathrm{P}=0.11)$.
In group 1, HDL cholesterol values did not differ between the basal test and the 3-month $(\mathrm{P}=0.80), 12$-month $(\mathrm{P}=0.95)$ and 24-month ( $\mathrm{P}=0.44)$ evaluations. Moreover, no difference in HDL values emerged between group 1 and group 2 at 12 months $(\mathrm{P}=$ 0.09 ) or 24 months $(\mathrm{P}=0.09)$.

LDL values were significantly higher at the baseline than at 3 months ( $\mathrm{P}=0.009), 12$ months $(\mathrm{P}<0.0001), 24$ months ( $\mathrm{P}$ $=0.001$ ) and 36 months $(\mathrm{P}=0.009)$ in group 1 (Figure 4); and also between group 1 and group 2 at 12 months $(\mathrm{P}=0.02)$ and 24 months $(\mathrm{P}=0.002$ ) (Figure 5). In group 2, by contrast, the difference in LDL values between the basal test and the 24-month assessment was not significant $(\mathrm{P}=0.82)$.

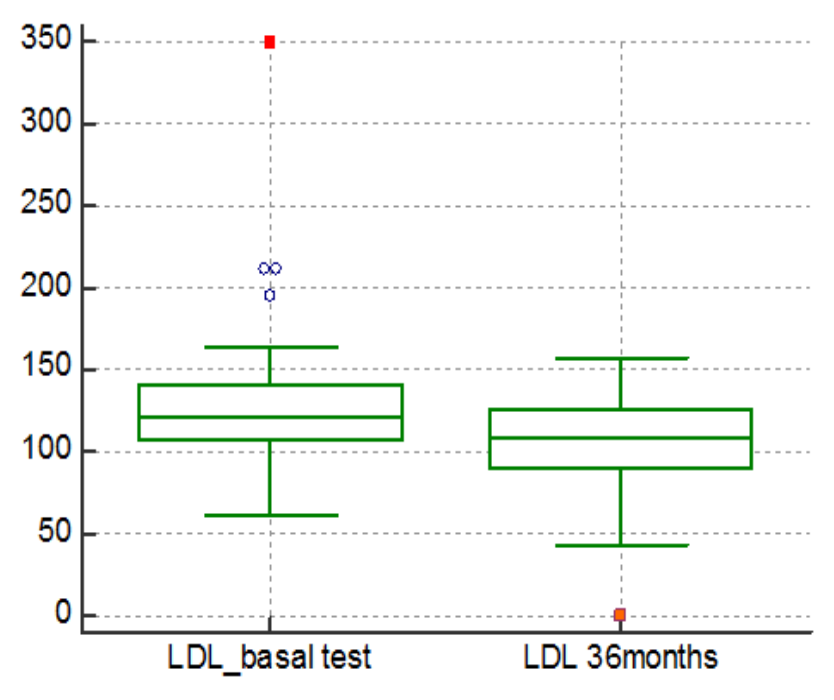

Figure 4: Differences in LDL-cholesterol between basal test and 36 month evaluation in group 1

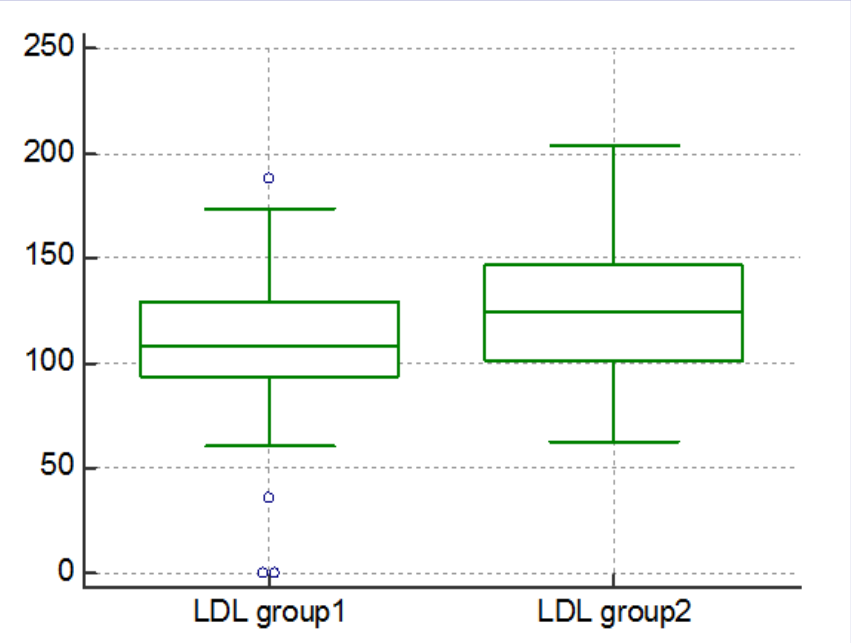

Figure 5: Differences in LDL-cholesterol between group 1 and group 2 at 12 months evaluation

\section{Discussion and Conclusions}

In our DTC patients in whom metformin was initiated, there was a significant reduction in TSH from the baseline evaluation to the 3- and 12-month evaluations, with a concomitant reduction 
in BMI. At 24 months, however, BMI was comparable to the basal values. The group in which metformin was initiated presented a higher baseline value of TSH than the control group, the difference being clearly significant $(P=0.0009)$. At the 12 -month evaluation, TSH remained higher in group 1 than in group 2, though the significance of the difference was clearly lower $(\mathrm{P}=$ 0.02). However, the 24-month evaluation showed no significant difference in TSH between the two groups, though in concomitance with a greater dosage of LT4 in group $1(\mathrm{P}=0.02)$. In group 1 patients, in whom metformin was started, the reduction of TSH at 3 and 12 months seems justified by the concomitant reduction in BMI. The potential ability of metformin to interfere with TSH values was first hypothesized by Virgersky et al. [20], who reported lower TSH levels on metformin, without modification of F-T3 and F-T4 values and without an obvious clinical effect. In a larger population, Fourier et al. [21] found a TSH reduction in diabetic patients taking metformin, without association with clinical parameters. A TSH reduction has also been reported in patients treated with L-T4, but not in euthyroid patients. Capelli et al. [14] observed a reduction in TSH only in the case of TSH levels $>2.5 \mathrm{mIU} / \mathrm{L}$. This reduction was not dependent on BMI or on the presence/absence of thyroperoxidase antibodies (TPOAb). Interestingly, TSH is not modified in patients in subclinical hyperthyroidism [22] with TSH levels similar to our population.

Our data showed no significant differences in the doses of RAI administered (which could be a reflection of the size of the carcinoma) to patients, nor were significant differences seen in thyroglobulin, which is the most reliable marker of disease progression in DTC when no interfering antibodies are present $[17,23]$. Klubo-Gwiezdzinska et al. [10], however, observed that tumours were smaller on diagnosis in DTC patients on metformin, than in diabetic DTC patients who had never been treated with metformin and non-diabetic DTC patients. In the study by Capelli et al. [14] no adverse events were recorded in diabetic patients treated with an average dosage of metformin of $1750 \mathrm{mg} /$ day. The occurrence of adverse events in our experience, however, seems to limit the therapeutic use of metformin in DTC, as diarrhoea and other gastrointestinal symptoms (20\%) led to withdrawal of the drug. However, we cannot exclude the possibility that poor patient compliance and the lack of conviction of the general practitioner, despite the information received on the role of off-label metformin in non-diabetic DTC, favoured drop-out at the first adverse symptom. Weight reduction is a favourable anecdotal finding after the initiation of metformin. In the study by Mousavi et al. [4], BMI did not differ between DTC patients on Metformin and those on placebo; however, after a 33\% reduction in L-T4 dosage, a weight increase was seen only in patients on placebo. In our population, the BMI of patients in whom metformin was initiated (group 1) was higher than that of control patients (group 2) at the baseline evaluation; this difference was maintained, despite the start of metformin, with comparable statistical significance. It is known that dysmetabolic alterations are an important co-morbidity in DTC patients [24]. Our data show that metformin determines a significant improvement in lipid status in DTC patients. Hormones and cytokines derived from adipose tissue, together with insulin resistance, have been implicated in the higher frequency of the DTC in obesity [25].
Thus, improving the lipid status in DTC by means of metformin may have a long-term protective role against the recurrence of cancer. From the metabolic point of view, the UKPDS has shown that metformin reduces cardiovascular events in overweight diabetic patients. Moreover, in a population of patients with type 2 diabetes mellitus who were not on lipid-lowering therapy, Lin et al. [26] found that metformin reduced LDL cholesterol levels. In our study, this effect was confirmed: a reduction in LDL and total cholesterol was recorded in group 1 during the three evaluations, and between group 1 and group 2(control subjects); this suggests that metformin reduces cardiovascular risk by lowering LDL cholesterol without the need to increase lipid-lowering therapies). In addition, in our study, metformin, as expected on account of its anti-diabetic action, reduced the levels of haemoglobin glycated over the three evaluations in group 1 and in the comparison between group 1 and group 2 at 12 and 24 months. However, the data on fasting blood glucose, HOMA index and insulinemia appear to be inconclusive. In DTC, some effects of a possible sub-clinical hyperthyroidism have not been thoroughly investigated. Indeed, metformin treatment in DTC could initially cause cardiovascular-related events, including an increase in the known risk of cardiac arrhythmia, typically atrial fibrillation [27, 28]. In our study of patients on suppressive and semi-suppressive L-T4 dosages, but rarely in therapeutic hyperthyroxinaemia, we did not observe changes in parameters of cardiovascular function after the initiation of metformin. Moreover, in a sub-set of diabetic euthyroid patients in whom metformin was started, and in whom TSH decreased steadily over six months to a level $<0.4 \mathrm{mU} / \mathrm{L}$, Capelli et al. [29] did not observe changes in PA, heart rate or ECG parameters. Our prospective study of diabetic DTC patients on metformin and of DTC patients without metformin therapy seems to confirm the relative safety of metformin from the cardiovascular point of view and to indicate that there is no need for a preventive reduction in the dosage of L-T4. In conclusion, our prospective data confirm the favourable evolution of DTC and document the high number of DTC patients with obesity and dysmetabolism. It is difficult to undertake metformin treatment in non-diabetic DTC patients, owing to problems of compliance and adverse events, and, according to our data, its effects seem to be beneficial only on the lipid status. In our group of patients with DTC and DMT2, no significant differences emerged in terms of Tg reduction or in terms of reduced RAI activity. Since in in vivo experimental models, metformin has been seen to reduce the size of the induced thyroid tumour [10], we cannot exclude the possibility that a longer observation time and a larger number of patients may be needed in order to obtain conclusive results. Similar conclusions emerged from a meta-analysis [30] on the effect of metformin on TSH in vivo. This meta-analysis, however, considered a small number of studies $(n=7)$ involving only 206 patients. Indeed, the conclusions of such studies are also limited by the difficulty of excluding all possible bias between treated subjects and controls, by the lack of detailed information on the dosage of metformin, which is often not reported, by the short duration of treatments (often less than 12 months) by the heterogeneity of clinical records, the absence of multicentre studies and the frequent lack of detailed data on TSH and metabolic control. Indeed, in their 2017 review, Meng et al. also 
agree that the effect of metformin on thyroid function and on DTC requires further studies on larger populations. In agreement with Lupoli et al. [30] and Meng et al. [31], we can therefore affirm that, in order to avoid confounding factors and to obtain convincing results, prospective studies of representative patients should be conducted. These should involve homogeneous subsets of disease, and, if possible be multi-centre.

The present study has the most numerous DTC population without DM in whom metformin has been started as off-label therapy. It would be interesting to study patients on metformin over much longer times, since DTC can relapse even 10-15 years after onset and primary treatments.

\section{Declarations}

\section{Ethics approval and consent to participate}

The study protocol was presented to the Ethics Committee of the Policlinico San Martino of Genoa, and all participating patients provided written informed consent.

\section{Consent for publication}

All patients provided written consent to publish their data anonymously

\section{Availability of data and material}

The patient's data used to support the findings of this study are available from the corresponding author upon request

\section{Competing Interests}

The authors declares that there is no conflict of interest regarding the publication of this paper.

\section{Abbreviations}

DTC: Differentiated thyroid carcinoma

DMT2: Type 2 diabetes mellitus

Tg: $\quad$ thyroglobulin

AMPK: AMPc-dependent protein kinase

TSH: thyrotropin

TgAb: anti-Tg antibodies

f-T3: free T3

f-T4: free T4

RAI: radioactive iodine

LT4: levothyroxine

BMI: body mass index

ATA: American Thyroid Association

RAI: radioactive iodine

HOMA: Homeostasis Model Assessment

\section{References}

1. Haugen BR, Alexander EK, Bible KC, Doherty GM, Mandel SJ, Nikiforov YE, et al. 2015 American Thyroid Association Management Guideline for adult patient with thyroid nodules and differentiated thyroid cancer. Thyroid. 2016;26(1):1-133. doi: 10.1089/thy.2015.0020

2. Kasznicki J, Sliwinska A, Drzewoski J. Metformin in cancer prevention and therapy. Ann Transl Med. 2014;2(6):57. doi: 10.3978/j.issn.23055839.2014.06.01

3. Shyang-Rong Shih, Wei-Yih Chiu, Tien-Chun Chang, Chin-Hsiao Tseng. Diabetes and thyroid cancer risk: literature review. Experimental Diabetes Research. 2012;2012:1-7. doi:10.1155/2012/578285

4. Mousavi Z, Dourandish L, Rokni H, Sadeghi R, Rasoul Zakavi S. Effects of short-term metformin therapy associated with levothyroxinee dose decrement on TSH and thyroid hormone levels in patients with thyroid cancer. Minerva Endocrinol. 2014;39(1):59-65.

5. Romero R, Erez O, Hüttemann M, Maymon E, Panaitescu B, CondeAgudelo A, et al. Metformin, the aspirin of the 21st century: its role in gestational diabetes mellitus, prevention of preeclampsia and cancer, and the promotion of longevity. Am J Obstet Gynecol. 2017;217(3):282302. doi: 10.1016/j.ajog.2017.06.003

6. Foretz M, Guigas B, Bertrand L, Pollak M, Viollet B. Metformin: from mechanisms of action to therapeis. Cell Metab. 2014;20(6):953-966. doi: 10.1016/j.cmet.2014.09.018

7. Madiraju AK, Erion DM, Rahimi Y, Zhang XM, Braddock DT, Albright RA.Metformin suppresses gluconeogenesis by inhibiting mitochondrial glycerophosphate dehydrogenase.Nature. 2014;510(7506):542-546. doi: $10.1038 /$ nature 13270

8. Safe S, Nair V, Karki K. Metformin-induced anticancer activities: recent insights. Biol Chem. 2018;399(4):321-335. doi: 10.1515/hsz-20170271

9. Salani B, Del Rio A, Marini C, Sambuceti G, Cordera R, Maggi D. Metformin, cancer and glucose metabolism. Endocr Relat Cancer. 2014;21(6):R461-471. doi: 10.1530/ERC-14-0284

10. Klubo-Gwiezdzinska J, Costello Jr J, Patel A, Bauer A, Jensen K, Mete M, et al. Treatment with metformin is associated with higher remission rate in diabetic patients with thyroid cancer. J Clin Endocrinol Metab.2013; 98(8): 3269-3279. doi: 10.1210/jc.2012-3799

11. Jang EK, Kim WG, Kwon H, Choi YM, Jeon MJ, Kim TY, et al. Metformin Is Associated with a Favorable Outcome in Diabetic Patients with Cervical Lymph Node Metastasis of Differentiated Thyroid Cancer. Eur Thyroid J. 2015; 4(3):181-188. doi: 10.1159/000437365

12.Tseng C-H. Metformin reduces thyroid cancer risk in Taiwanese patients with type 2 diabetes. PLoS One. 2014;9(10): e109852. doi: 10.1371/journal.pone.0109852

13. Pappa $\mathrm{T}$ and Alevizaki M. Metformin and Thyroid: an update. Eur Thyroid J 2013;2:22-28. doi: 10. 1159 /00 0346248

14. Cappelli C, Rotondi M, Pirola I, Agosti B, Formenti A, Zarra E, et al. Thyreotropin Levels in diabetic patients on metformin treatment. Eur J Endocrinol. 2012;167(2):261-265. doi: 10.1530/EJE-12-0225

15. Krysiak R and Okopien B. The effect of metformin on the hypothalamicpituitary-thyroid axis in women with polycystic ovary syndrome and subclinical hypothyroidism. J Clin Pharmacol. 2015;55(1):45-49. doi: 10. 1002 / jcph.373 
16. Al-Alusi MA, Du L, Li N, Yeh MW, He X, Braverman LE, et al. Metformin does not suppress serum thyrotropin by increasing levothyroxine absorption. Thyroid. 2015;25(10):1080-1084. doi: 10.1089/ thy.2015.0211

17. Giusti M, Zoccola R, Guazzini B, Molinari E, Valenti S, Villa G, et al. Recombinant human TSH changes the multidisciplinary approach to patients with differentiated thyroid carcinoma, Minerva Endocrinol. 2003; 28(3):191-203.

18. Friedewald WT, Lewy RI, Fredrickson DS. Estimation of the concentration of low-density lipoprotein cholesterolo in plasma, without use of the preparative ultracentrifuge. Clin Chem.1972; 18(6):499-502.

19. Matthews DR, Hosker JP, Rudenski AS, Naylor BA, Treacher DF, Turner RC. Homeostasis model assessment: insulin resistance and beta-cell function from fasting plasma glucose and insulin concentrations in man. Diabetologia 1985;28(7):412-419.

20.Vigersky RA, Filmore-Nassar A, Glass AR. Thyrotropin suppression by metformin. J Clin Endocrinol Metab. 2006;91(1):225-227.

21. Fournier J.P, Yin H, Hoi Yun Yu O, Azoulay L. Metformin and low levels of thyroid-stimulating hormone in patients with type 2 diabetes mellitus. CMAJ. 2014;186(5):1138-1145. doi: 10.1503/cmaj.140688

22. Krysiak R, Szkrobka W, Okopien B. The effect of metformin on the hypothalamic-pituitary-thyroid axis in patients with type 2 diabetes and subclinical hyperthyroidism. Exp Clin Endocrinol Diabetes. 2015 ;123(4):205-208. doi: 10.1055/s-0034-1398621

23. F. Pacini, F. Basolo, R. Bellantone, G. Boni, M. A. Cannizzaro, M. De Palma. Italian consensus on diagnosis and treatment of differentiated thyroid cancer: joint statements of six Italian societies. J Endocrinol Invest. 2018;1-28. doi:10.1007/s40618-018-0884-2
24. Giusti M, Mortara L, Degradi R, Cecoli F, Mussap M, Rogriguez G, et al. Metabolic and cardiovascular risk in patients with a history of differentiated thyroid carcinoma: A case-controlled cohort study. Thyroid Res. 2008 ; 1(1):2. doi: 10.1186/1756-6614-1-2

25. Pappa T and Alevizaki M. Obesity and thyroid cancer: A clinical update. Thyroid. 2014;24(2):190-199. doi: 10.1089/thy.2013.0232

26. Lin S.H, Cheng P.C, Tu S.T, Hsu S.R, Cheng Y.C, Liu Y.H. Effect of metformin monotherapy on serum lipid profile in statin-naïve individuals with newly diagnosed type 2 diabetes mellitus: a cohort study. PeerJ. 2018;6:e4578. doi: 10.7717/peerj.4578

27. Portella RB, Pedrosa RC, Coeli CM, Buescu A, Vaisan M. Altered cardiovascular vagal responses in nonelderly female patients with subclinical hyperthyroidism and no apparent cardiovascular disease. Clin Endocrinol (Oxf). 2007;67(2):290-294.

28. Petretta M, Bonaduce D, Spinelli L, Vicario ML, Nuzzo V, Marciano F, et al . Cardiovascular haemodinamics and cardiac autonomic control in patients wiyh subclinical and overt hypertiroidism. Eur J Endocrinol. 2001;145(6):691-696

29. Cappelli C, Rotondi M, Pirola I, Agosti B, Formenti A, De Cata P, et al. Metformin-induced thyrotropin suppression is not associated with cardiac effects. Hormones (Athens). 2014;13(2):252-258.

30. Lupoli R, Di Minno A, Tortora A, Ambrosio P, Lupoli G.A, Di Minno M.N.D. Effects of Treatment With Metformin on TSH Levels: A Meta-analysis of Literature Studies. J Clin Endocrinol Metab. 2014; 99(1):E143-E148. doi: 10.1210/jc.2013-2965

31. Meng X, Xu S, Chen G, Derwahl M, Liu C. Metformin and thyroid disease. J Endocrinol. 2017;233(1):R43-R51. doi: 10.1530/JOE-16-0450 